\title{
A Personal Appreciation of Walter Noll
}

\author{
A. Ian Murdoch ${ }^{1}$
}

Received: 19 April 2018 / Published online: 30 October 2018

(C) The Author(s) 2018

\begin{abstract}
Walter Noll is personally acknowledged to have been an inspirational teacher and an exemplar in his analysis of fundamental concepts and relations in Continuum Mechanics. Attention is drawn to unfounded criticisms of two aspects of his work and how considerations of local spatial and temporal averaging of microscopic behaviour can complement and inform the continuum viewpoint. Remarks on such considerations briefly summarise the results of published works which both utilised Noll's direct notation and attempted to emulate his precision and clarity.
\end{abstract}

Keywords Reminiscences $\cdot$ Criticisms $\cdot$ Microscopic considerations

Mathematics Subject Classification $70 \cdot 74 \mathrm{~A}$

\section{Reminiscences}

The inspiration and expertise of teachers and mentors form an invaluable basis for the development of any student. In this respect I was singularly fortunate to begin graduate studies in the Mathematics Department of Carnegie-Mellon University in the Autumn of 1970. Most significantly, the following Spring I took a first course in Continuum Mechanics from Mort Gurtin and was immediately attracted to the subject and stimulated by the enthusiasm and rigour with which he presented material. Prior to arriving in the Department I had been unaware of the subject, and that two of its leading exponents, Walter Noll and Bernard Coleman, were among its members. Walter was held in the highest regard by students and, inter alia, his development of direct notation ${ }^{1}$ influenced a course I took from Bill Williams

\footnotetext{
${ }^{1}$ That is, at no stage are co-ordinates employed, but geometrical points (and displacements between pairs of such points) are treated directly.

A.I. Murdoch

a.i.murdoch@maths.strath.ac.uk

1 Department of Mathematics and Statistics, University of Strathclyde, Livingstone Tower, 26 Richmond Street, Glasgow, G1 1XH, UK
} 
on Multidimensional Algebra and Calculus in my first semester. However, it was only in Spring 1972 that I experienced Walter first-hand, sitting in on a course which formed the basis of his expository memoir [1]. It is not easy to convey the impact of his lectures to anyone who has only read his works and thus missed the careful motivation behind their content. In crystal clear fashion he discussed fundamental continuum concepts and relations in terms of axioms and a notation free of superfluous elements. His calm and measured delivery was precise, and gave a lasting impression of someone who had not only thought deeply about every item, but who also instinctively thought logically. Here was someone one might aspire to emulate, although his habit of smoking cigarillos is now no longer an option! Both in and out of class Walter was approachable and, when answering any of my queries, always displayed a level of understanding deeper than my own, no matter how long or hard I had considered the points in question. On a personal level Walter was always kind, calm, courteous, and in my experience never lost his temper nor disparaged the work of others.

Prior to undertaking doctoral research it was necessary to pass an oral comprehensive examination in two subjects. Mine took place in September 1972 with choices Continuum Mechanics and Complex Analysis, and examiners Walter, David Owen and Zeev Nehari. In preparation I had studied the derivation of the stress tensor beyond the level of coursework, and in some detail, but was not satisfied with my understanding. Passing Walter on a corridor some days before the exam, I confessed this apologetically. He replied that he did not understand either! ${ }^{2}$ In retrospect I guess it was only natural that, after being asked to present something of my choice for ten minutes (I spoke on the notions of observers and Euclidean point spaces), Walter asked me to talk about the stress tensor. Fortunately I managed to say something sensible. Having enjoyed (and passed) this exam I needed to sort out a doctoral supervisor. Somewhat intimidated by Walter's reputation, and guided by the grapevine of senior fellow graduate students, I was fortunate enough to be accepted as a student by Mort Gurtin. The following March, the 15th meeting of the Society for Natural Philosophy (addressing mathematical problems related to mixtures of interacting species) was held at Rochester, NY. As Chairman, Walter had expenses paid by the Society, but opted to stay with friends, and most kindly donated his hotel room etc to myself (otherwise beyond my means). This was both surprising and deeply appreciated, particularly since I knew nothing of the subject matter and was very much a rookie. It is still a pleasure to recall my first meeting with such luminaries as Truesdell and Müller.

By this time, after first having me look into time-reversal considerations for materials with internal state variables, Mort had suggested that my $\mathrm{PhD}$ topic should be 'surfaces', having noted that I had taken Walter's course in Differential Geometry in the Fall of 1972. Here I understood that Mort was thinking of surfaces of bodies, and my first reaction was to think about liquid boundaries and surface tension. The next day I queried whether he meant interfaces, since any material boundary such as that between two immiscible liquids may exhibit properties which depend upon both liquids. Receiving the answer 'I guess so' led to my consideration of bidimensional continua sandwiched between pairs of bulk phases. Here Walter's notes on surfaces proved invaluable by virtue of their clear geometric visualisations, which involve local bijections between surface points and corresponding tangent planes, and are expressed in direct notation. While it was necessary to develop a theory of bidimensional bodies ('material surfaces') via definitions of configurations, deformations, and delineation of appropriate divergence theorems for vector and tensor fields defined on surfaces, these were mainly direct analogues of standard three-dimensional concepts, and perceived as such via use of Walter's notation. The resulting thesis, owing significantly to Mort's guidance

\footnotetext{
${ }^{2}$ Stress remained one of his interests over the years: cf. [2-4].
} 
and meticulous input, was examined by Professors Gurtin, Markovitz, Noll, and Williams, and published verbatim as [5]. Later work on symmetry considerations for elastic material surfaces [6,7] (of both second-grade and Cosserat varieties) was likewise greatly indebted to Walter's approach.

\section{Unfounded Criticisms}

\subsection{Preamble}

Most researchers will never have been fortunate enough to have heard Walter either teach or talk, and be directly acquainted with his work and ideas only via his papers or articles. The concise nature of these cannot always convey viewpoint, motivation or reasoning behind his particular approach. ${ }^{3}$ While the value and depth of his papers were widely advertised by his former adviser Clifford Truesdell, this sometimes proved to be less helpful than intended. Truesdell's writings were stimulating and greatly informative, but often their confrontational stance could alienate researchers with different scientific cultural backgrounds; here one only has to think of understandable responses to his designation of 'rational' mechanics in respect of his favoured approaches. Unfortunately, as a consequence of such alienation, and lack of acquaintance with contemporary mathematical concepts and notation, Noll's work has not been fully appreciated. In several cases criticism has come from within the Continuum Mechanics fraternity.

\subsection{Simple Fluids}

Rivlin took issue with Noll's definition of a simple fluid. Noll (cf. [9, 10]) had shown that the stress response functional for any simple fluid can be expressed via material frameindifference $^{4}(\mathrm{mfi})$ in terms of a reduced functional whose domain consists only of positivedefinite symmetric tensor histories which at current time take the value $\mathbf{1}$, the identity tensor. The restriction imposed by mfi at this stage is that such reduced functional be invariant under the orthogonal group. Rivlin [11] argued that the definition of simple fluid for the functional in question mandated invariance under the unimodular group, a stronger restriction than that of Noll, given the larger nature of the unimodular group. However, such restriction for Rivlin involved histories which at current time take the form $\mathbf{H}^{T} \mathbf{H}$ with $\mathbf{H}$ unimodular. Since $\mathbf{H}^{T} \mathbf{H}=\mathbf{1}$ if and only if $\mathbf{H}$ is orthogonal, this criticism is unfounded: such histories do not lie in the domain of the reduced response functional unless $\mathbf{H}$ is orthogonal. This was pointed out by myself in [12] and the source of the error identified. This error stemmed from a failure to distinguish between the domain of a functional and that which corresponds to a reduced form of this functional. Rivlin did not accept this technicality and replied with a repetition of his mistake $[13,14]$. This proved to be a salutary lesson in having to appreciate that, for whatever reason, one cannot always convince someone of their misconceptions or errors. Such a situation, of much greater significance, also arose concerning the status and interpretation of mfi.

\footnotetext{
${ }^{3}$ Perhaps with this in mind Walter drafted the collection of essays [8] and established the website www.math. cmu.edu/ wn0g/noll.

${ }^{4}$ Introduced by Truesdell and Noll in [10] as a principle of classical physics and variously phrased on pages 36,44 and 296 therein.
} 


\subsection{Material Frame-Indifference}

In a summer school held in 1982 at Noto, Sicily, I learned from Ingo Müller of his approximative constitutive relations for stress and heat flux fields in a rarefied gas derived on the basis of kinetic theory (cf. [15]). These he claimed to violate mfi because these fields were dependent upon spin, contradicting the impossibility of such dependence as argued in most texts on the basis of mfi (cf., e.g., [16], p. 156, [17], p. 251). However, what such texts accomplish is to rule out dependence upon spin as computed relative to the frame of a general observer. Crucially, however, Müller's spin is computed with respect to an (and hence any) inertial frame ${ }^{5}$ and accordingly was a field upon which all observers could agree, since the notion of inertial frame is central to classical physics (in serving to establish the concept of force). However, from my understanding of mfi as expounded in Noll's lectures, such dependence upon inertial spin was indeed possible, and this I indicated to Ingo. Since he strongly disagreed, I thought long and hard on the matter and wrote [18] to explain my perspective. I also wrote to Walter to see if I had misunderstood the nature of mfi. In his reply of November, 1983, Walter wrote 'As to the matter concerning frame-indifference, I fully agree with your viewpoint, and I told Ingo Müller so when I recently met him at a meeting in Providence, RI.' Unfortunately Ingo never accepted this and the nature of mfi/objectivity continues to be controversial, particularly if it is identified with 'invariance under superposed rigid motions' (isrbm). However, the possibility that material response could be sensitive to spin relative to an inertial frame was accepted by both Noll and Müller and does not violate any fundamental tenet of Physics.

\subsection{Comments on MFI, ISRBM and Spin-Dependence}

The essence of the notions of $\mathrm{mfi}$ and isrbm stems from recognition that constitutive relations which purport to describe material behaviour as manifest to different observers must be subject to some restriction in order to make sense. Said differently, any two observers should be able to agree upon the nature and behaviour of any given material of interest, and to recognise how this imposes restrictions upon, and relations between, response functions. How to label, frame and phrase discussion of this fundamental aspect of modelling has long been problematic. As detailed on pp. 28-29 of [19], over the years Walter variously described the concept as 'isotropy of space' and 'objectivity' before finally settling on 'frame-indifference'.

It clarifies matters to discard once and for all the isrbm interpretation. While at first sight it is 'obvious' that rotation of a body without distortion will merely rotate (inter alia) the stress field therein in precisely the same way, a little thought suggests this might not be the case. If the body is initially at rest in an inertial frame, then subsequent rotation with respect to this frame will involve a motion which introduces non-uniform acceleration of points of the body. Accordingly, some form of external force system is required to preserve rigidity. Just how this might be achieved is problematic, not to say metaphysical. Of course, what isrbm immediately achieves by its very nature is to negate any constitutive dependence upon spin since no restriction is placed upon the rate at which rotation is performed. Since material behaviour rarely seems to exhibit spin dependence, its absence might be regarded as evidence of the veracity of isrbm. However, fundamental considerations indicate that spin dependence is to be expected quite generally: common experience merely indicates

\footnotetext{
${ }^{5}$ This was not entirely clear from the paper, but subsequently clarified via correspondence with, and correction by, the author.
} 
that sensitivity thereto is usually negligible. To appreciate this somewhat subtle point, one must recognise that continuum fields describe physical behaviour which is monitored by measuring devices. In order to interpret recorded data it is necessary to relate localised measurement values to continuum field values. However, local measurements involve associated scales of both length and time, since no measuring device can register physical information that is either instantaneous or localised at a geometrical point. ${ }^{6}$ Rather, measurement values represent local space-time averages of material behaviour at specific scales. Thus any measurement made on a body must be expected to be sensitive to rotation of the body (which occurs during the time lapse required to register any given measurement) relative to the measuring device employed. ${ }^{7}$ Accordingly, fields such as stress and heat flux must a priori be expected to be sensitive ${ }^{8}$ to rotation with respect to an (hence any) inertial frame. In [15] Müller had exhibited such sensitivity via kinetic theory. The foregoing considerations should suffice to put paid to isrbm, but it has proved a hard beast to slay and continues to attract believers.

It was helpful to talk privately with Rivlin, at Nottingham in 1985, on the matter of isrbm. In a few words he disposed of its standard interpretation by saying that of course one should not envisage rigidly rotating a body of interest relative to the observer, but rather rotating the observer relative to the body. Although such motions would appear identical to the observer (consider films taken of the body with a camera held by the observer in which any 'background' has been erased: both films reveal the same relative rotation) there is a physical difference between the two relative rotations. The state of the body has not changed as a consequence of rotation of the observer (for whom field values will merely be perceived to be rotated versions of those which correspond to absence of relative rotation). Rivlin [23] described this viewpoint as 'invariance under superposed rotation'. In practice this is almost identical with 'invariance under change of observer' (cf. [24], p. 206): here a change of observer, from $O$ to $O^{*}$ say, is envisaged in which the same response functions are employed by $O$ and $O^{*}$. This notion may be re-interpreted as a change in perspective of a single observer were he/she to have undergone a rotation. In so doing there can be no query (otherwise reasonable) as to why the same response functions should be involved: for example, in the case of elastic bodies, just why $O$ and $O^{*}$ should of necessity select the same reference configuration becomes immediately apparent.

Since isrbm continued to be regarded widely as an absolute statement, in 2002 I wrote [25] to point out that it was helpful to emphasise the nature of objectivity in its broad sense as stemming from observer consensus. Here I was influenced by comments made by Jerry Ericksen, in a letter of November, 1980, when discussing a model of heat conduction which I felt illuminated the isrbm/mfi controversy (cf. (2) in [26]): 'As I interpret the word, objectivity refers to what it is that different observers can agree upon. First, different observers can and do make their own choice of reference configuration'. However, I was ignoring the advice of Truesdell who, in October, 1980, wrote "I hope you can avoid using "objectivity" because that term is often used (e.g. by Müller) to denote "invariance under superposed rigid motions".' In [25] attention was drawn to observer consensus implicit in usage of mathematical ideas and results, to physical concepts such as inertial frames, events, and, for classical

\footnotetext{
${ }^{6}$ For example, a 'snapshot' has an associated length scale of 'resolution' and a time scale of 'exposure'.

${ }^{7}$ Such device should be at rest in some inertial frame to obviate its own possible sensitivity to rotation.

${ }^{8}$ Of course, this sensitivity may be very slight, and detectable only at high spins. However, the point is that it is to be expected and is in the very nature of things. Indeed, such sensitivity has been suggested in studies of superfluid Helium [20, 21] and small-scale fluid turbulence [22]. Models which interpret large-scale measurements of atmospheric air movement or oceanographic currents would also seem to be candidates for inclusion of constitutive dependency upon inertial spin.
} 
physics, to time lapses and distances between simultaneous events. The specific additional aspects of objectivity for continuum modelling of macroscopic behaviour were postulated to be: agreement upon the nature of any given ideal material, and upon all possible responses of an ideal material, no matter what the relative motion of the observers might be. Derivation of restrictions upon response functions involved precisely Rivlin's notion of behaviour conceived in terms of two separate motions of a single observer relative to the body. The argumentation employed a second observer in order to stress that different observers may choose different response functions, echoing Ericksen's above remark.

A more detailed discussion which includes microscopic considerations is to be found in Chap. 12 of [27].

\section{Influence}

Noll's legacy derives not only from his works but also from the example set by the precision and clarity he brought to the understanding, formulation and presentation of fundamental aspects of continuum modelling. In attempting to extend or complement his work, it is the latter that has been of most personal interest. Specifically, I have tried to examine the physical basis of primitive objects which appear in Noll's axiomatic approach and the general continuum viewpoint.

It is usual to regard a body to be a set of material points which at any instant occupy a definite region in space. While this may appear to be entirely natural, courtesy of our sensory perception of matter at the macroscopic level, Nature is more complex, and can require much finer investigation for its character to be revealed. Since macroscopic material properties ultimately derive from the behaviour of molecules, which in turn consist of assemblies of atomic nuclei and electrons, it is natural to explore how the macroscopic/continuum viewpoint can be related to discrete/microscopic considerations. If one starts from a model of a system of interacting point masses, it is necessary to implement an averaging procedure applied to discrete entities in order to establish fields which can be identified with those of continuum physics. This can be done via either adopting the ensemble averaging of Statistical Mechanics followed by further spatial (and possible temporal) averaging or direct application of spatial (with possible additional temporal) averaging. ${ }^{9}$ The results of thirty years thinking about such issues form the content of [27]. The discussions therein are couched in direct notation and employ Noll's notion of each observer perceiving space in terms of an individual copy of Euclidean space: such considerations are addressed in detail in two appendices. Local spatial averaging of corpuscular behaviour is implemented via scale-dependent weighting functions, and results in fields that may be averaged further in time. Bodies (regarded as systems of point masses) whose corpuscular content change with time are treated via the notion of a membership function $e$ : at any given time a point mass may belong to the system $(e=1)$ or not $(e=0)$. Such considerations lead to an appreciation of the physical interpretations of fields, and the relations they satisfy, which is deeper than those which appear intuitively clear from a macroscopic viewpoint (wherein the material of which a body consists at any instant is regarded to be smeared throughout a definite portion of space that it is considered to occupy). Some consequences of the foregoing microscopic viewpoint are now briefly outlined in a sequence of remarks. These remarks are intended to stimulate interest in study of the cited works.

\footnotetext{
${ }^{9}$ Cf., e.g., p. 821 of Irving and Kirkwood's classic contribution [28], [29] and Sect. 14.5 of [27].
} 
Remark 1 Spatial densities of mass $\rho$ and momentum $\mathbf{p}$ at any prescribed length scale $\varepsilon$ are defined in terms of a weighting function $w$ and satisfy (via a simple calculation: cf. [27], pp. 46-47) the continuity equation

$$
\partial \rho / \partial t+\operatorname{div} \mathbf{p}=0
$$

While the form (1) is independent of the specific form of $w$, the smoothness of fields defined thereby are inherited from that of $w$ and the physical interpretations thereof depend upon the choice of $w$. The simplest choice yields the values of $\rho$ and $\mathbf{p}$ at location $\mathbf{x}$ and time $t$ as the total mass and momentum of the point masses within a distance $\varepsilon$ of $\mathbf{x}$ at time $t$ divided by $4 \pi \varepsilon^{3} / 3$.

Remark 2 The region occupied by the body at scale $\varepsilon$ is that in which $\rho>0$ and is thus, for the simplest choice of $w$, the union of a set of spherical regions each of radius $\varepsilon$ and centred at a body point mass. The boundary of this region (to be regarded as the boundary of the body at this scale) is accordingly scale-dependent. This is helpful when modelling porous media: at sufficiently large scale pores are not evident, but at decreasing scales pore boundaries become increasingly well-resolved, and pore space increases. Porosity is then a spatial average, at large scale, of pore space defined in terms of the smaller scale, and is accordingly a concept which involves two length scales (cf. [27], Remark 4.3.5). It can be useful to consider two porosities, defined via selection of two different small scales, since the region available to any permeating fluid will depend on both its molecular size and phase (liquid or gas).

Remark 3 Since $\rho$ and $\mathbf{p}$ are scale-dependent (see Remark 1) the velocity

$$
\mathbf{v}:=\mathbf{p} / \rho
$$

is also scale-dependent (cf. [27], Sect. 3.6). From Remark 1 it follows that the simplest choice of $w$ yields $\mathbf{v}(\mathbf{x}, t)$ as the velocity of the mass centre of point masses within the radius $\varepsilon$ spherical averaging region centred at $\mathbf{x}$ at time $t$. The corresponding motion map, with respect to the situation at any selected reference time, is defined in terms of the solution to an initial-value problem. Roughly speaking, this corresponds to consideration of any point in the region occupied by the body at the reference time and subsequently moving from this point with a velocity which at any later time coincides with the instantaneous local value of $\mathbf{v}$. With each point of the reference-time region can be identified a material point. This notion is thus seen to be a mathematical artefact whose motion is governed by $\mathbf{v}$ (and hence is scale-dependent) and, for the simplest choice of $w$, represents a local mass centre velocity. Such visualisation renders a precise physical interpretation of a concept which is usually undefined, or loosely described in terms of 'marks on a body'. The scale-dependence of $\mathbf{v}$ upon context is exemplified by attempting to describe motion of air as it flows over an aerofoil, round a skyscraper, or in meteorological contexts.

Remark 4 The motion of a point mass (which represents any fundamental discrete entity such as a molecule, atom or ion) in an inertial frame is governed by Newton's second law in which account is taken of interactions therewith from all other molecules of the body and also from external agencies. Spatial averaging of such equations of motion ${ }^{10}$ yields directly

\footnotetext{
${ }^{10}$ Each equation of motion is multiplied by the weighting function evaluated at field point $\mathbf{x}$ and time $t$ for the point mass in question and the sum of such equations is taken over all point masses.
} 
the local form (cf. [27], Sect. 5.5)

$$
\mathbf{f}+\mathbf{b}=\partial / \partial t\{\mathbf{p}\}+\operatorname{div} \mathcal{D}
$$

of linear momentum balance. Here $\mathbf{f}$ denotes the interaction force density and $\mathbf{b}$ the resultant external force density ( $\mathbf{b}=\rho \mathbf{g}$ if gravity is the only such agency). Tensor field $\mathcal{D}$ takes symmetric values and involves thermal velocities. ${ }^{11}$ Any tensor $\mathbf{T}^{-}$(termed the interaction stress tensor) which satisfies

$$
\operatorname{div} \mathbf{T}^{-}=\mathbf{f}
$$

yields ${ }^{12}$ the standard local form of balance

$$
\operatorname{div} \mathbf{T}+\mathbf{b}=\rho \mathbf{a} \quad \text { with } \mathbf{T}:=\mathbf{T}^{-}-\mathcal{D} .
$$

Here $\mathbf{T}$ denotes the Cauchy stress tensor and is seen to have two distinct contributions. For ideal gases, in which interactions are absent (so $\mathbf{T}^{-}=\mathbf{0}$ ), $-\mathcal{D}$ constitutes a pressure.

Remark 5 In treating non-reacting mixtures the analogue of (5) for a species $\alpha$ is (cf. [27], Sects. 9.3-5)

$$
\operatorname{div} \mathbf{T}_{\alpha}+\mathbf{f}_{\alpha}+\mathbf{b}_{\alpha}=\rho_{\alpha} \mathbf{a}_{\alpha} \quad \text { with } \mathbf{T}_{\alpha}:=\mathbf{T}_{\alpha}^{-}-\mathcal{D}_{\alpha} .
$$

Here partial stress $\mathbf{T}_{\alpha}^{-}$involves only $\alpha-\alpha$ interactions, $\mathbf{f}_{\alpha}$ represents a body force density which derives from the effect of all other constituents upon species $\alpha$, and $\mathbf{b}_{\alpha}$ is the resultant external body force density for this species. These interpretations are immediate, and differ from those postulated in early works by Truesdell and others on mixtures (cf., e.g., [30, 31]) which were incorrect and led to a paradox, as pointed out by Gurtin, Oliver \& Williams [32].

Remark 6 Spatial averaging of the moments of individual equations of motion about an arbitrary point yields a local moment of momentum balance, and averaging applied to the scalar product of each such equation with the relevant corpuscular velocity leads to a local form of energy balance (cf. [27], Ch. 7 and 6, respectively). Here thermal quantities are distinguished by the presence of thermal velocities in their definitions. For interactions delivered in terms of separation-dependent pair potentials, the internal energy density is the sum of densities of energy of assembly and heat content. ${ }^{13}$

Remark 7 Additional balances can be derived by averaging moments of mass, and tensor moments of individual equations of motion about an arbitrary point. These balances involve a local measure of fine-scale inhomogeneity ${ }^{14}$ together with couple stress, and, if adopted, require appropriate book-keeping in derivation of the (modified) energy balance (cf. [27], Ch. 6, 7).

\footnotetext{
${ }^{11}$ That is, velocities of point masses relative to the local continuum velocity $\mathbf{v}$.

${ }^{12}$ Of course, the physical/geometric interpretation of $\mathbf{T}^{-}$depends upon which solution to (4) is chosen (cf. [27], Sect. 5.8).

${ }^{13}$ That is, kinetic energy associated with thermal motions.

${ }^{14}$ For the simplest form of weighting function, this measure at $(\mathbf{x}, t)$ is the displacement from $\mathbf{x}$ of the mass centre associated with those point masses in the sphere centred at $\mathbf{x}$ with radius $\varepsilon$ at time $t$.
} 
Remark 8 Further averaging in time, at any temporal scale $\Delta$ (essentially a mean value over a time interval of duration $\Delta$ ending at current time $t$ ), is straightforward and balances remain formally unchanged (cf. [27], Ch. 8). Of course, field values are now identified as local averages jointly in space and time at prescribed scales $\varepsilon$ and $\Delta$, and as such have the same nature as measurement values. In computing any such value, at location $\mathbf{x}$ and time $t$, time averaging is effected keeping $\mathbf{x}$ fixed, and so, if this value is to be identified with a local measurement, then this measurement corresponds to a fixed point for the observer. This makes sense for measuring devices which deliver local information but remain stationary relative to the observer. Temporal averages taken over material point trajectories (and which thus yield information upon which all observers can agree) requires further study.

Remark 9 Any material system $M$ whose microscopic content changes with time ${ }^{15}$ (and accordingly exchanges matter with its environment) can be treated in terms of a membership function $^{16} e$. When applied to reacting mixtures, the considerations of Remark 3 make it clear how material points which correspond to different constituents can 'occupy' the same locations, and how material points for a given constituent can suddenly vanish or appear (cf. [27], Ch. 8).

Remark 10 Modelling electrons and atomic nuclei as point mass/charges, spatial averaging can be used to establish Maxwell's equations as they pertain to electrostatics and magnetostatics [33] and thereby elucidate the character of electromagnetic fields.

Over the years attempts to master concepts, arguments, and technicalities have incurred many instances of personal frustration and no little nervous tension. At such times it has always been helpful to pause, take stock, and ask myself: How would Walter have approached the problem? A profound legacy indeed.

Publisher's Note Springer Nature remains neutral with regard to jurisdictional claims in published maps and institutional affiliations.

Open Access This article is distributed under the terms of the Creative Commons Attribution 4.0 International License (http://creativecommons.org/licenses/by/4.0/), which permits unrestricted use, distribution, and reproduction in any medium, provided you give appropriate credit to the original author(s) and the source, provide a link to the Creative Commons license, and indicate if changes were made.

\section{References}

1. Noll, W.: Lectures on the foundations of continuum mechanics and thermodynamics. Arch. Ration. Mech. Anal. 52, 62-92 (1973)

2. Noll, W.: Die Herleitung der Grundgleichungen der Thermomechanik der Kontinua aus der statistischen Mechanik. J. Ration. Mech. Anal. 4, 627-646 (1955)

3. Noll, W.: On the theory of surface interactions (2005), 14 pp., published on the website www.math.cmu. edu/ wnOg/noll

4. Noll, W.: Thoughts on the concept of stress. J. Elast. 100, 25-32 (2010)

5. Gurtin, M.E., Murdoch, A.I.: A continuum theory of elastic material surfaces. Arch. Ration. Mech. Anal. 57, 291-323 (1975)

\footnotetext{
${ }^{15}$ E.g. A creature or vehicle which gains propulsion by ejection of fluid, constituent in a reacting mixture, or the set of molecules which define the exposed surface of a propagating crack.

${ }^{16}$ See text preceding Remark 1.
} 
6. Murdoch, A.I., Cohen, H.: Symmetry considerations for material surfaces. Arch. Ration. Mech. Anal. 72, 61-98 (1979)

7. Murdoch, A.I., Cohen, H.: Symmetry considerations for material surfaces: addendum. Arch. Ration. Mech. Anal. 76, 393-400 (1981)

8. Noll, W.: Contributions to Natural Philosophy. Research Report 04-CAN-0187, Center for Nonlinear Analysis, Carnegie-Mellon University (2004)

9. Noll, W.: A mathematical theory of the mechanical behaviour of continuous media. Arch. Ration. Mech. Anal. 2, 197-226 (1958)

10. Truesdell, C., Noll, W.: The non-linear field theories of mechanics. In: Flügge, S. (ed.) Handbuch der Physik III/3. Springer, Berlin (1965)

11. Rivlin, R.S.: A note on the simple fluid. J. Non-Newton. Fluid Mech. 11, 209-213 (1982)

12. Murdoch, A.I.: On unfounded criticism of the simple fluid of Noll. J. Non-Newton. Fluid Mech. 12, 387-392 (1983)

13. Rivlin, R.S., Smith, G.F.: On the fallacy in a paper by A.I. Murdoch. J. Non-Newton. Fluid Mech. 12, 393-394 (1983)

14. Murdoch, A.I.: Reply to the note "On the fallacy in a paper by A.I. Murdoch" by R.S. Rivlin and G.F. Smith. J. Non-Newton. Fluid Mech. 15, 247-248 (1984)

15. Müller, I.: On the frame dependence of stress and heat flux. Arch. Ration. Mech. Anal. 45, 241-250 (1973)

16. Silhavy, M.: The Mechanics and Thermomechanics of Continuous Media. Springer, Berlin (1997)

17. Gurtin, M.E., Fried, E., Anand, L.: The Mechanics and Thermodynamics of Continua. Cambridge University Press, Cambridge (2010)

18. Murdoch, A.I.: On material frame-indifference, intrinsic spin, and certain constitutive relations motivated by the kinetic theory of gases. Arch. Ration. Mech. Anal. 83, 185-194 (1983)

19. Noll, W.: The genesis of Truesdell's nonlinear field theories of mechanics. J. Elast. 70, 23-30 (2003)

20. Roberts, P.H., Donnelly, R.J.: Superfluid mechanics. In: Van Dyke, M., Vincentini, W.G., Wehausen, J.V. (eds.) Annual Review of Fluid Mechanics, vol. 6. Annual Reviews, Palo Alto (1974)

21. Hills, R.N., Roberts, P.H.: Superfluid mechanics for a high density of vortex lines. Arch. Ration. Mech. Anal. 66, 43-71 (1977)

22. Piquet, J.: Turbulent Flows, revised 2nd printing edn. Springer, Berlin (2001)

23. Rivlin, R.S.: Red herrings and sundry unidentified fish in nonlinear continuum mechanics. In: Kanninen, M.G., Adler, D., Rosenfield, A.R., Jaffee, R.I. (eds.) Inelastic Behaviour of Solids, pp. 117-134. McGraw Hill, New York (1970)

24. Jog, C.S.: Continuum Mechanics, 3rd edn. Cambridge University Press, Cambridge (2015)

25. Murdoch, A.I.: Objectivity in classical continuum physics: a rationale for discarding the "principle of invariance under superposed rigid body motions' in favour of purely objective considerations. Contin. Mech. Thermodyn. 15, 309-320 (2003)

26. Murdoch, A.I.: On material frame-indifference. Proc. R. Soc. Lond. A 380, 417-426 (1983)

27. Murdoch, A.I.: Physical Foundations of Continuum Mechanics. Cambridge University Press, Cambridge (2012)

28. Irving, J.H., Kirkwood, J.: The statistical mechanical theory of transport processes. IV. The equations of hydrodynamics. J. Chem. Phys. 18, 817-829 (1950)

29. Murdoch, A.I.: Some primitive concepts in continuum mechanics regarded in terms of objective spacetime averaging: the key role played by inertial observers. J. Elast. 84, 69-97 (2006)

30. Truesdell, C.: Rational Thermodynamics. McGraw Hill, New York (1969)

31. Bowen, R.M.: Theory of mixtures. In: Eringen, A.C. (ed.) Continuum Physics III. Academic Press, New York (1976)

32. Gurtin, M.E., Oliver, M.L., Williams, W.O.: On balance of forces for mixtures. Q. Appl. Math. 30, 527530 (1973)

33. Murdoch, A.I.: On spatially-averaged electrokinetics of point charges and Maxwell's equations. J. Elast. 131, 75-109 (2018) 